\title{
Dynamic Label Assignment for End-to-End Object Detection
}

\author{
Feizhao Zhang, Qingling Zhang*, Zewen Mo \\ Sun Yat-sen University, Shenzhen 518107, Guangdong, China \\ *Correspondence Author, zhangqling@mail.sysu.edu.cn
}

\begin{abstract}
In the design of object detection algorithms, the label Assignment and positive/negative sample identification are very important, affecting the convergence speed and object detection performance. Nowadays, positive/negative sample identification mainly focuses on one-to-many label assignment of Ground-Truth and Bounding Box, and few researchers have paid attention to the label assignment of end-to-end object detection models. In this paper, aiming at end-to-end object detection models, we propose a dynamic label assignment algorithm based on the Hungarian matching method, and normalization methods of L1 loss at the stage of cost calculation and loss calculation. We test the proposed dynamic label assignment algorithm included the end-to-end model SparseRCNN with the COCO dataset. The resulting AP achieved an improvement of 0.4 when we train the model on four GPUs and an improvement of 0.6 when training the model on a single GPU.
\end{abstract}

Keywords: Computer vision, Deep learning, Object detection, Label assignment, Improved algorithms.

\section{Introduction}

Object detection [1] is the basis of many computer vision applications, such as instance segmentation, image caption, object tracking, etc. Object detection recognizes the category and localizes the position of objects that humans care about in pictures and videos with computers. With the rapid development of deep learning techniques, object detection has been rejuvenated, attracting unprecedented attention $[2,3,8$, 9]. Consequently, it has been widely applied to many realworld applications, such as self-driving, robot vision, video surveillance, etc [39, 40, 41].

Modern object detection models based on convolutional neural networks (CNN) can be grouped into three categories according to prediction methods: dense detection $[2,3,4,5,6$, 7], dense-to-sparse detection [8, 9, 10, 11], and sparse detection $[12,13,14,20]$. However, whether it is a dense detection or a sparse detection, one or more most appropriate anchor boxes or the location of the grid cell [38] will be first allocated to a certain Ground-Truth (GT) for the following prediction process. For a given GT, the selection of an anchor or a grid cell is a very crucial step in object detection, which is known as label assignment.

For label assignment, a GT or background is generally assigned to an anchor box or a grid cell according to predesigned rules. The anchor box or the grid cell assigned to a GT is called a positive sample and will participate in the calculation of loss in subsequent classification and regression steps. The anchor box or the grid cell assigned to a background is called a negative sample, and will only participate in the loss calculation of the classification step. RetinaNet [4] adopts the Intersection-over-Union (IoU) between the GT and a corresponding anchor box as the basis for assigning labels. FCOS [15] without anchor boxes identifies positive and negative samples by checking whether a grid cell falls into the center area of a GT. ATSS [5] applies a strategy based on both to obtain statistical characteristics of anchor boxes and grid cells, and then assign labels. Other studies [16, 17, 18] report that the confidence score of each anchor box can be used as an indicator in a dynamic assigning strategy, and anchor boxes with higher confidence can be easily learned by an object detection model. Experiments demonstrate that these label assignment strategies can achieve state-of-the-art performance.

OTA [19] argues that the method of identifying positive and negative samples without considering the context might be sub-optimal. because it heavily relies on manually designed rules when dealing with anchor boxes that can be assigned to multiple GTs. OTA formulates label assignment as the Optimal Transportation (OT) problem and defines GT (including the background) as a supplier that can provide a certain number of labels, and then defines each anchor box as a demander that needs a unique label. And the number of anchor boxes assigned to each GT is dynamically calculated according to a global anchor box regression state, leading to ambiguous boxes to be utilized.

The above label assignments apply a one-to-many strategy, i.e. one GT will be assigned to multiple anchor boxes, and a corresponding object detection network needs a nonmaximum suppression (NMS) to filter out redundant Bounding Boxes (BBoxes) due to replicated anchor boxes in the predicted results. Nevertheless, such a hand-crafted algorithm can cause severe performance drop in some cases (e.g. dense scenes). Detection Transformer [12] (DETR) is the first algorithm that attempts to consider label assignment from a global perspective and is also the first sparse prediction object detection network that applies transformer [21] to computer vision. In terms of label assignment, it utilizes the Hungarian method to make a one-to-one label match according to the cost matrix of each query and calculates all GTs globally. Thus, end-to-end object detection without anchor boxes and post-processing can be realized. Nonetheless, the label assignment process in DETR is fixed in the whole training process, unlike OTA's dynamic adjustment, and cannot be adaptively changed in accordance with each stage during model training, leading to low accuracy and poor convergence performance (detailed analysis is shown in the main text).

In this paper, we propose a novel dynamic label assignment method with consideration of the Hungarian algorithm. We also argue that the model should have a different emphasis on 
classification and regression tasks at different training stages and confirm its effectiveness. Therefore, the process of label assignment can be adjusted dynamically. We empirically found that classification accuracy will increase promptly in the initial stage of model training when most samples are easy to classify. Accordingly, the network ought to mine hard examples and propose more samples that are difficult to classify [4]. In our algorithm, we reshape the matching cost matrix in the Hungarian algorithm that plays the same role as label assignment in former object detection models. The weight of $\mathrm{L} 1$ cost will be lowered in the cost of BBoxes whose generalized IoU (giou) [22] with GT is greater than the threshold value of $\alpha$, in order to enable the model to refine the BBoxes with a better regression prediction quality. Due to the reduced cost of regression, samples that are not classified as optimal may have the chance to be selected as positive samples at this step, to mine samples that are hard to classify. During training, we set the giou cost at -1 for BBoxes whose giou with a GT is greater than $\beta$. It is obvious that the BBoxes whose giou with a GT greater than $\beta$ are high-quality regression samples, and in the middle and later stages of training, we will get many high-quality regression anchor boxes. As a result, we give more attention to the classification accuracy of these samples, so that the model can finally predict high-accuracy and high-quality BBoxes.

We also analyze and discuss the four prediction parameters of the BBoxes by using GT or image when calculating the L1 term in cost and loss.

Finally, the proposed algorithm is included into some end-toend object detection models and then be tested on the MSCOCO dataset [23]. Without increasing the amount of computation and training time, the performance has been improved obviously under the conditions of a single GPU and Multi-GPU training.

\section{Related Work}

\subsection{One-to-Many Label Assignment}

It is a pivotal procedure for object detection models to assign a GT or a background to an anchor box. One-to-many label assignment means that one GT might be allocated to multiple anchor boxes. For an anchor-based object detection model, a certain IoU between anchor boxes and their corresponding GT is usually used as the allocation criterion. For example, Region Proposal Network (RPN) of Faster R-CNN [8] defines anchor boxes with IoU greater than 0.7 as positive samples and those with IoU less than 0.3 as negative samples. This stage is thus a dense prediction. At the second stage of a sparse prediction, the IoU threshold for positive/negative division is changed to 0.5 , and each anchor box will select the GT whose IoU is the maximum among all IoUs greater than 0.5 as the label, which is thus called the Max IoU matching rule. This rule has been proved to be very effective and has been adopted by many variants $[9,24,25,26,27,28]$ of Faster R-CNN as well as many one-stage object detection algorithms $[29,30,2$, $31,4]$.

All of the above object detection models are anchor-based detectors. The anchor-free models which are generally single- stage prediction models show high computational efficiency and simplicity, without calculating IoUs for a large number of anchor boxes. These models assign labels to the grid cells of the feature maps. CornerNet [32] determines the position of the BBoxes through the corner points of the prediction boxes. The GT is mapped to the feature map, and it is stipulated that a predicted grid cell of the feature map falls within a Gaussian kernel region of the GT mapping point, and the closer to the mapping point the more likely the grid cell to be a positive sample. FCOS [15] does not predict the offset of grid points, but directly predicts the distance between the grid cells and four sides of the GT. If a grid cell falls into the central areas of multiple GTs at the same time, the GT with the smallest area is selected as the label, and the grid cell is marked as a positive sample.

The above detectors adopt pre-designed rules (such as the threshold of IoU or a certain area of grid cells) to assign labels to the preset anchor boxes or the grid cells of the feature map. However, such an algorithm may not be optimal for more complicated cases, and only relying on the regression state to define positive and negative samples may cause inconsistency between regression and classification.

\subsection{One-to-One Label Assignment}

One-to-one label matching algorithms usually discard NMS post-processing, thus avoiding the problems caused by NMS. CenterNet [20] uses keypoint estimation to solve the object detection problem, and predicts the offset of the center point and the size for a BBox. Because each object has only one corresponding center point, post-processing is not required. The rule of label assignment is similar to CornerNet [32], but only one prediction point is selected as a positive sample for the object. DETR [12] uses the classification and regression of prediction as the cost matrix of the Hungarian algorithm for bipartite matching and finally obtains the prediction result without post-processing. POTO [33] assumes that there is still a gap between the sample selected by the cost of the Hungarian algorithm and the loss desired by the model, so the cost is redesigned by using the spatial prior and the weighted geometric mean of classification and regression. However, it still suffers relatively lower performance, compared to the one-to-many object detection models with post-processing.

We found that the problem of label matching method used in current end-to-end models, such as DETR [12], Deformable DETR [13], and SparseRCNN [14], etc., is consistent with the viewpoint put forward by OTA [19]. The whole training process adopts a completely fixed assignment method. As a result, the model might not achieve optimal convergence, and detailed analysis on this will be presented the Section 3.2. At different training stages, classification and regression must be emphasized differently and adjusted dynamically. Finally, we introduce the label assignment algorithm with dynamic adjustment.

\section{Methodology}

\subsection{Rethinking Box Normalization}

Currently, the GT and BBox are normalized in the calculation 
of the cost and the loss in end-to-end object detection algorithms based on the Hungarian algorithm. Mathematically, it can be expressed:

$$
\left\{\begin{array}{c}
x_{l}^{t_{n}}=\frac{x_{l}^{t}}{w_{i m g}}, x_{l}^{b_{n}}=\frac{x_{l}^{b}}{w_{i m g}} \\
y_{l}^{t_{n}}=\frac{y_{l}^{t}}{h_{i m g}}, x_{l}^{b_{n}}=\frac{y_{l}^{b}}{h_{i m g}} \\
x_{r}^{t_{n}}=\frac{x_{r}^{t}}{w_{i m g}}, x_{r}^{b_{n}}=\frac{x_{l}^{b}}{w_{i m g}} \\
x_{r}^{t_{n}}=\frac{y_{r}^{t}}{h_{i m g}}, x_{r}^{b_{n}}=\frac{y_{l}^{b}}{h_{i m g}}
\end{array}\right.
$$

where $x^{t}$ and $x^{b}$ are GT and BBox before normalization respectively. $x^{t_{n}}$ and $x^{b_{n}}$ are GT and BBox after normalization respectively. $l$ indicates the upper left corner of the BBox and $r$ indicates the upper left corner of the BBox. $h_{i m g}$ and $w_{i m g}$ are the height and width of an image. In (1), $h_{i m g}$ and $w_{i m g}$ are used for normalization (img-norm).

From the above definition, we can see the difference between the GT and the BBox after normalization is still absolute, which is equivalent to multiplying the cost and the loss by a weight, taking no account of the scale of BBoxes.

Consequently, it cannot reflect the real regression situation of multi-scales BBoxes leading to the drop of AP.

To solve the above drawback, we consider that the image normalization can be replaced by the GT normalization (gtnorm). It offers two important advantages:1) when the relevant parameters of a GT are used to normalize the BBox, it reflects the error percentage. 2) It can express the regression state of the multi-scales box. As shown in Figure 1, the error is 0.4 using gt-norm, and 0.0625 using img-norm (the default height and width of the image are both 640). The former is more consistent with the error calculated with IoU.

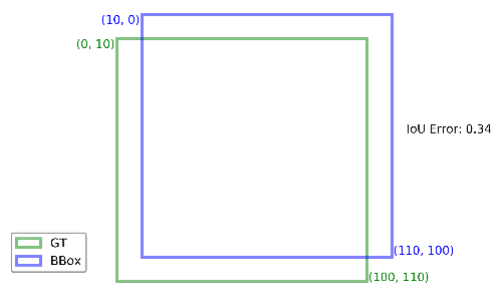

Figure 1: IoU Error (error $=1-\mathrm{IoU})$

\subsection{Reset the Weight}

Since the magnitude of gt-norm is larger than that of imgnorm, we will redesign the weights of the cost and the loss. For that purpose, we first examine the cost and loss computed by classification and regression under different goodness of fit. For classification, we calculate the change of focal cost and focal loss in the range 0.0 to 1.0 . For regression, we set the giou in accordance with the BBox with different percentage errors (the error range is 0.0 to 1.0 ), so as to obtain the cost and loss under different giou error conditions. We sum the L1 distance of the four coordinate parameters normalized by GT as the L1 cost and L1 loss. Based on the above exploration, we redesign the classification and regression from the weight of the original papers $(2,5,2)$ and tried to find the best proportion combination.

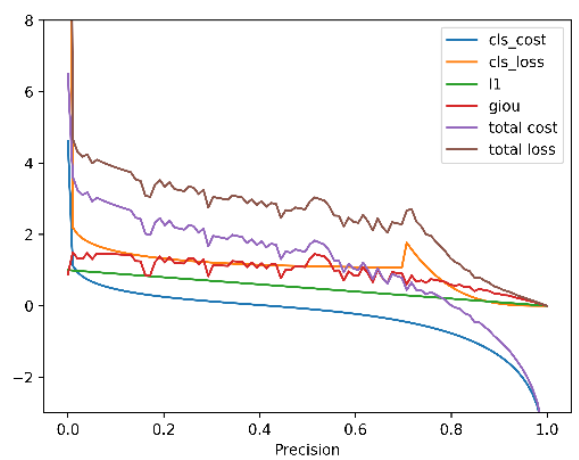

Figure 2: The curve of cost and loss with precision

In terms of cost calculating, there is little difference in classification cost if a focal cost is used at the early and middle steps of training. For example, when the probability of positive samples is between 0.4 and 0.6 , there is little difference in the cost, and the overall range of change is not between 0.0 and 1.0 (Figure 2). Therefore Linear Classification Cost (LCC) is designed as the following formula:

$$
\operatorname{Cost}_{c l s}=1-p
$$

where $p$ is the probability of the query and Cost $_{c l s}$ is the cost of classification, the variation range of which is mapped between 0.0 and 1.0 .
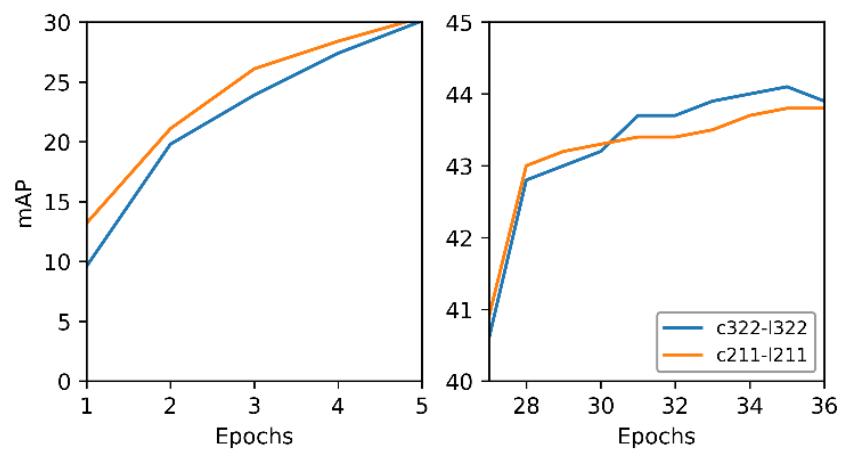

Figure 3: The curve of mAP. c322 denotes that the weight of cost is set to $3: 2: 2$. 1322 denotes that the weight of loss is set to $3: 2: 2$.

We test various types of weights based on the LCC and gtnorm. It is obvious that when the weight of classification cost increases, a model can reach a faster convergence at the early training stage (Figure 3). While in the later periods, due to insufficient high-quality samples, a high-precision regression cannot be achieved, and therefore we cannot get the best training model. On the other hand, if we increase the weight of regression, AP at the early stages will be lower, because more positive samples are judged as negative samples, and the regression at the later stages can obtain better results. But the best model can still not be achieved, because the classification accuracy of the model is not at a high level. 


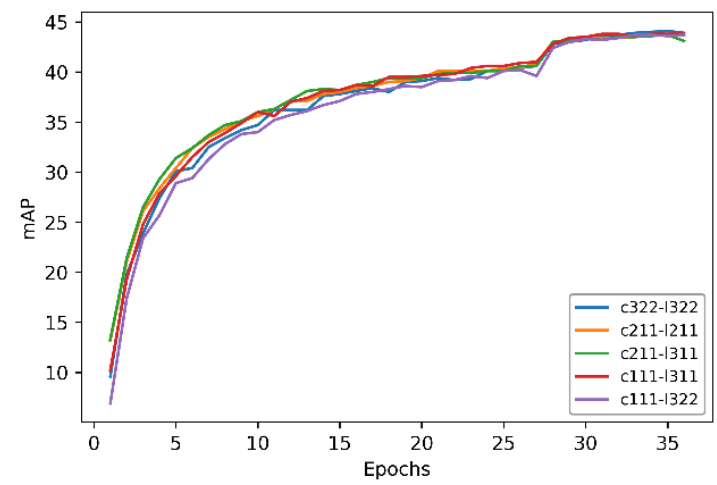

Figure 4: The curve of mAP with different weights.

Through experiments, we found that setting the cost ratio of classification and regression to $1: 1: 1$ or $3: 2: 2$ can achieve better results, with a corresponding loss ratio of $3: 1: 1$ or $3: 2: 2$. From Figure 4, it can be inferred that the model training is more sensitive to the setting of cost weights. Meanwhile, when all weights are fixed, LCC is replaced by Focal Classification Cost (using Focal loss to calculate the cost of all positive sample classes, FCC). The FCC is sensitive to samples with either low accuracy or high accuracy.
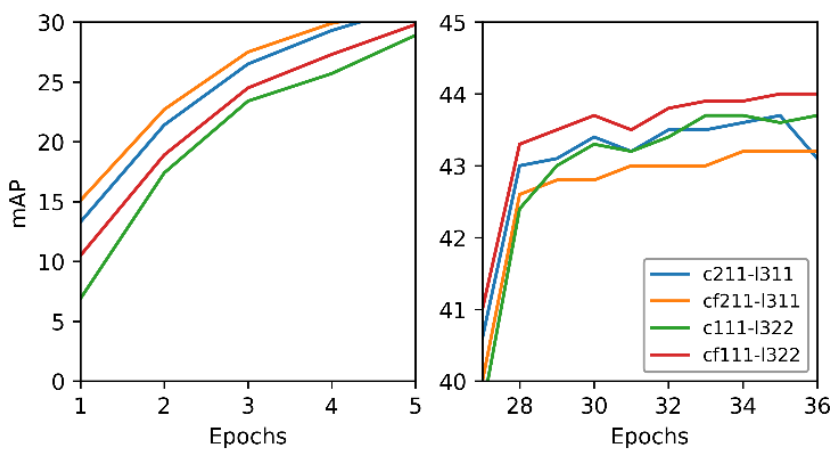

Figure 5: The curve of mAP with different classification costs The cf211 denotes that the classification of cost is FCC.

It can be deduced from Figure 5 that, under the same weight, the early convergence rate of FCC is faster than that of LCC, and the final result is almost better than that of LCC. FCC is sensitive to samples with high accuracy so that it can improve the model's ability to achieve high-accuracy classification. In addition, from the comparison of the two groups of experiments, we advocate that the weight of classification in cost and loss cannot be too high for FCC, because setting higher weight and FCC properties will lead to excessive neglect of regression tasks.

Based on the above analysis, we can conclude that classification and regression weights cannot both be set too large. Cost is more likely to be affected by the weight than loss and then lead to the distinct detection ability of a model. FCC performs better than LCC, because it can provide samples that are difficult to classify for learning at the early stages of training, and accelerate the convergence of the model. At the later stages of training, it can enable the model to focus on the high-accuracy classification.

\subsection{Redesign the Cost}

In an end-to-end object detection algorithm model, the
Hungarian algorithm plays the role of selecting positive and negative samples, and the cost represents the comprehensive situation of BBox classification and regression in selecting positive and negative samples. Therefore, the calculation of cost and the corresponding weight setting of cost are vitally important, since it determines the samples that the model should pay more attention to at each stage during the training process.

We now know that FCC performs better than LCC, and we also know that using gt-norm for normalization can better reflect the BBox regression state. Consequently, we can select positive and negative samples based on a real regression state.

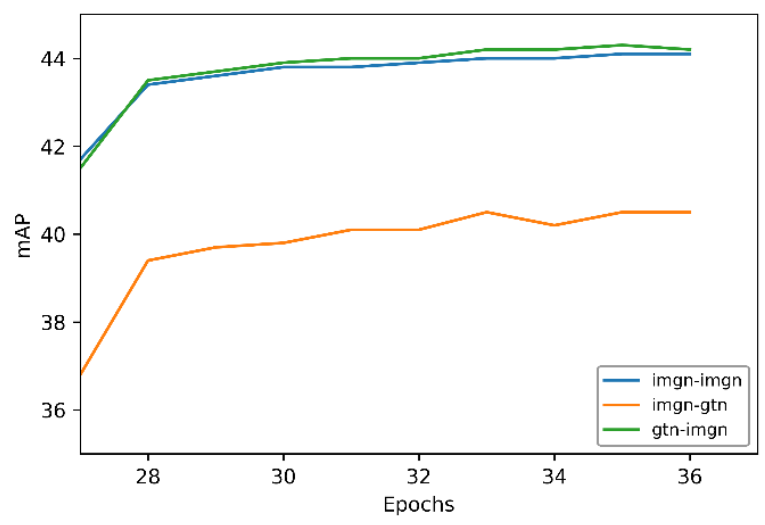

Figure 6: The comparison of img-norm and gt-norm. The imgn and gtn denote img-norm and gt-norm respectively. The gtn-imgn means the former (gt-norm) for the cost stage and the latter for the loss stage.

It can be inferred from Figure 6 that using GT for normalization can achieve better results. The principle is that IoU can basically be considered as the overlap rate of the BBox and the GT, and the error obtained by utilizing GT for normalization can be regarded as the deviation rate between the heights and the width of BBox and GT. Therefore, its evaluation criteria are consistent with IoU. Using it as the selection standard is more conducive to ensure high-quality samples be chosen at the training stage and for the model regression. However, if the GT is also used to calculate the loss, it will not have an identical effect. This is because when the size of the GT is 800 and the error of the BBox is 80 , or when the size of the GT is 400 and the error of the BBox is 40 , the losses are 0.1 in both cases, which creates tolerance for larger-sized objects, and is adverse to the high-quality regression of such objects. Therefore, for loss calculation we adopt the img-norm to ensure high-quality regression for large-sized objects.

According to the above analysis, we designed a method to dynamically assign positive and negative samples, in which the cost caused by the errors of giou and L1 is adjusted dynamically. The specific formula is as the following:

$$
\begin{aligned}
& \text { Cost }_{\text {giou }}=\left\{\begin{array}{cc}
-w_{g} \times \text { giou, } & \text { when giou }<\alpha \\
-w_{g}, \quad \text { when giou } \geq & \alpha
\end{array}\right. \\
& \text { Cost }_{l 1}=\left\{\begin{array}{cc}
w_{l 1} \times \text { error }_{l 1}, & \text { when gio } \\
w_{l 1} \times \text { error }_{l 1} \times(1-\text { giou }), \text { when } & \text { giou } \geq \beta
\end{array}\right.
\end{aligned}
$$

where $\alpha$ and $\beta$ are hyperparameters, which are both 
empirically set to 0.7 . The design idea is that when the giou is greater than the presetting value, we will reduce the cost proportion of the regression part according to the regression state, and select the samples with higher classification accuracy from those with higher quality regression. In general, when the giou is greater than 0.7 , we consider it to be a highquality regression sample, and set the corresponding Cost $_{\text {giou }}$ to have no difference at the cost calculating stage. Meanwhile, the weights of $\operatorname{Cost}_{l 1}$ will be dynamically adjusted according to the value of the giou. On the other hand, for samples whose giou is less than 0.7 , we think they are lowquality regression samples, and pay more attention to their regression state rather than their accuracy of classification.

In fact, AP computed on the iou is widely used as the evaluation metric in many tasks. It is natural that giou can be considered as a reference for the regression state. We think that the weights of classification and regression parts in the cost should be dynamically adjusted according to the regression state of the box. In this paper, giou is regarded as the state of box regression, and the weights of Cost $_{\text {giou }}$ and $\operatorname{Cost}_{l 1}$ is dynamically adjusted by using the value of giou (that is, the weights of corresponding classifications are adjusted). Such dynamic label assignment strategy makes the high-quality samples with the same regression state focus on the improvement of classification accuracy, while the lowquality samples with different regression states take into account the classification and regression. At last, the overall accuracy of the model is improved.

\section{Experiments}

We use the MS-COCO 2017 object detection dataset [23] to validate our algorithm of dynamically selecting positive and negative samples for end-to-end models. All the models are trained on the COCO train2017 (about 118k images) and evaluated on the val2017 (5k images).

We conducted experiments on the efficient end-to-end object detector, namely the SparseRCNN, and the network settings were consistent with the original algorithm except for the selection of positive and negative samples. Specifically, as the following:

We opted for the ResNet-50 [34] as the backbone network, and the optimizer is the AdamW [35] with a 0.0001 weight decay. We carried out two different training: the first one is that the mini-batch is 4 images and all models are trained on a 3090 GPU; the second one is to train 16 images on 4 Tesla v100 GPUs. The training schedule is all 36 epochs, and the initial learning rate is set at $2.5 \times 10^{-5}$, which is divided by 10 at the 27 th epoch and the 33rd epoch respectively. The weight of the backbone is pre-trained by cosine annealing on the ImageNet [36], and other newly added layers are initialized with the Xavier [37]. The data augmentation used in the experiment includes horizontally flipping and randomly scaling the images while keeping the aspect ratio (the shorter side ranges from 480 to 800 , and the longer side is up to 1333).

We tried different combinations for the setting of hyperparameters $\alpha$ and $\beta$. As shown in Table 1 , when $\alpha$ and $\beta$ are set to 0.7 and 0.7 respectively, the model can obtain the best performance, with an AP of 45 . It can be concluded that when $\alpha$ and $\beta$ are increased, it will benefit medium and large objects and achieve a high-precision detection (which can be reflected through the variation of $\mathrm{AP}_{\mathrm{m}}$, $\mathrm{AP}_{1}$ and $\mathrm{AP}_{75}$ in Table 1 respectively). On the other hand, when $\alpha$ and $\beta$ are lowered, the $\mathrm{AP}$ of small objects $\left(\mathrm{AP}_{\mathrm{s}}\right)$ will be better.

Table 1: Analysis of different values of $\alpha$ and $\beta$

\begin{tabular}{cccccccc}
\hline$\alpha$ & $\beta$ & $\mathrm{AP}$ & $\mathrm{AP}_{50}$ & $\mathrm{AP}_{75}$ & $\mathrm{AP}_{\mathrm{s}}$ & $\mathrm{AP}_{\mathrm{m}}$ & $\mathrm{AP}_{1}$ \\
\hline 0.5 & 0.5 & 44.3 & $\mathbf{6 3 . 6}$ & 47.9 & $\mathbf{2 7 . 8}$ & 47.9 & 59.0 \\
0.5 & 0.7 & 44.0 & 63.4 & 47.7 & $\mathbf{2 7 . 8}$ & 47.5 & 58.0 \\
0.7 & 0.5 & 44.7 & 63.0 & 48.8 & 26.5 & 47.8 & $\mathbf{6 0 . 1}$ \\
0.7 & 0.7 & $\mathbf{4 5}$ & 62.6 & $\mathbf{4 9 . 1}$ & 27.2 & $\mathbf{4 8 . 8}$ & 59.7 \\
\hline
\end{tabular}

We trained the model on a 3090 GPU and the batch-size is 4, aiming at the cost calculation stage, the accuracy is compared by using the img-norm and the gt-norm (in which the weights of each part of classification and regression are set to $2: 5: 2$ and 1: 1: 1 respectively according to the conclusion of the Section 3.2). From Table 2 (The DLA denotes dynamic label assignment), it can be concluded that AP of the gt-norm is slightly improved, compared with the former baseline AP. Using the dynamic label assignment algorithm and img-norm, AP can be improved by 0.6 , compared with that reported in the original paper. Meanwhile, the strategy of dynamic label assignment and gt-norm enable the model to achieve 1.0 improvement on AP.

Table 2: The results of the model trained on a single GPU

\begin{tabular}{ccccccc}
\hline Method & $\mathrm{AP}$ & $\mathrm{AP}_{50}$ & $\mathrm{AP}_{75}$ & $\mathrm{AP}_{\mathrm{s}}$ & $\mathrm{AP}_{\mathrm{m}}$ & $\mathrm{AP}_{1}$ \\
\hline baseline & 44.1 & 63.2 & 47.9 & 27.0 & 47.2 & 59.0 \\
DLA+img-norm & 44.6 & $\mathbf{6 3 . 6}$ & 48.7 & $\mathbf{2 7 . 9}$ & 47.7 & 58.7 \\
DLA+gt-norm & $\mathbf{4 5}$ & 62.6 & $\mathbf{4 9 . 1}$ & 27.2 & $\mathbf{4 8 . 8}$ & $\mathbf{5 9 . 7}$ \\
\hline
\end{tabular}

The same model was trained on 4 Tesla V100 GPUs with 4 images per GPU, thus the total batch-size is 16 . As shown in Table 3, it can be concluded that the strategy of gt-norm achieved a slightly better performance. The dynamic label assignment with img-norm tactics, can achieve 0.3 improvement on AP. Furthermore, the combination of dynamic label assignment and gt-norm achieved 0.4 improvement on AP.

Table 3: The results trained on 4 GPUs

\begin{tabular}{ccccccc}
\hline Method & $\mathrm{AP}$ & $\mathrm{AP}_{50}$ & $\mathrm{AP}_{75}$ & $\mathrm{AP}_{\mathrm{s}}$ & $\mathrm{AP}_{\mathrm{m}}$ & $\mathrm{AP}_{1}$ \\
\hline baseline & 43.1 & 62.0 & 46.6 & 26.3 & 45.7 & 58.2 \\
DLA+img-norm & 43.4 & $\mathbf{6 2 . 0}$ & 46.7 & $\mathbf{2 7 . 0}$ & 46.3 & 58.0 \\
DLA+gt-norm & $\mathbf{4 3 . 5}$ & 61.6 & $\mathbf{4 7 . 5}$ & 25.5 & $\mathbf{4 7 . 1}$ & $\mathbf{5 9 . 1}$ \\
\hline
\end{tabular}

\section{Conclusion}

We proposed an algorithm to dynamically select positive and negative samples for the end-to-end models, and analyzed the performance of gt-norm and img-norm at the cost and loss calculation stages of end-to-end models. In conclusion, based on the strategy of dynamic label assignment, the performance of end-to-end models can be enhanced without increasing model parameters and basically without increasing extra computation cost by using the gt-norm at the cost calculation stage and the img-norm at the loss calculation stage. We hope that our work could provide a new option for the assignment of positive and negative samples in object detection 
algorithms, and inspire rethinking the relationship between label assignment at each stage of model training in object detection models.

\section{References}

[1] Z. Zou, Z. Shi, Y. Guo and J. Ye, "Object detection in 20 years: A survey" in arXiv:1905.05055v2, 2019, [Online] Available: https://arxiv.org/abs/1905.05055v2.

[2] Wei Liu, Dragomir Anguelov, Dumitru Erhan, Christian Szegedy, Scott Reed, Cheng-Yang Fu, and Alexander C Berg. Ssd: Single shot multibox detector. In European conference on computer vision, pages $21-37$. Springer, 2016.

[3] Joseph Redmon, Santosh Divvala, Ross Girshick, and Ali Farhadi. You only look once: Unified, real-time object detection. In Proceedings of the IEEE conference on computer vision and pattern recognition, pages 779 788, 2016.

[4] Tsung-Yi Lin, Priya Goyal, Ross Girshick, Kaiming He, and Piotr Dollar. Focal loss for dense object detection. In Proceedings of the IEEE international conference on computer vision, pages 2980-2988, 2017.

[5] Shifeng Zhang, Cheng Chi, Yongqiang Yao, Zhen Lei, and $\mathrm{Stan} \mathrm{Z} \mathrm{Li}$. Bridging the gap between anchor-based and anchor-free detection via adaptive training sample selection. In Proceedings of the IEEE/CVF Conference on Computer Vision and Pattern Recognition, pages 9759-9768, 2020.

[6] Han Qiu, Yuchen Ma, Zeming Li, Songtao Liu, and Jian Sun. Borderdet: Border feature for dense object detection. In European Conference on Computer Vision, pages 549-564. Springer, 2020.

[7] Lin Song, Yanwei Li, Zhengkai Jiang, Zeming Li, Hongbin Sun, Jian Sun, and Nanning Zheng. Finegrained dynamic head for object detection. arXiv preprint arXiv:2012.03519, 2020.

[8] Shaoqing Ren, Kaiming He, Ross Girshick, and Jian Sun. Faster r-cnn: Towards real-time object detection with region proposal networks. In Advances in neural information processing systems, pages 91-99, 2015

[9] Zhaowei Cai and Nuno Vasconcelos. Cascade R-CNN: Delving into high quality object detection. In CVPR, 2018.

[10] Jifeng Dai, Yi Li, Kaiming He, and Jian Sun. R-FCN: Object detection via region-based fully convolutional networks.In NeurIPS, 2016.

[11] Ross Girshick, Jeff Donahue, Trevor Darrell, and Jitendra Malik. Rich feature hierarchies for accurate object detection and semantic segmentation. In CVPR, 2014.

[12] Nicolas Carion, Francisco Massa, Gabriel Synnaeve, Nicolas Usunier, Alexander Kirillov, and Sergey Zagoruyko. End-to-end object detection with transformers. arXiv preprint arXiv:2005.12872, 2020.

[13] Xizhou Zhu, Weijie Su, Lewei Lu, Bin Li, Xiaogang Wang, and Jifeng Dai. Deformable detr: Deformable transformers for end-to-end object detection. arXiv preprint arXiv:2010.04159, 2020.

[14] Peize Sun, Rufeng Zhang, Yi Jiang, Tao Kong, Chenfeng Xu, Wei Zhan, Masayoshi Tomizuka, Lei Li, Zehuan Yuan, Changhu Wang, et al. Sparse r-cnn: End- to-end object detection with learnable proposals. arXiv preprint arXiv:2011.12450, 2020.

[15] Zhi Tian, Chunhua Shen, Hao Chen, and Tong He. Fcos: Fully convolutional one-stage object detection. In Proceedings of the IEEE international conference on computer vision, pages 9627-9636, 2019.

[16] Xiaosong Zhang, Fang Wan, Chang Liu, Rongrong Ji, and Qixiang Ye. Freeanchor: Learning to match anchors for visual object detection. In Advances in Neural Information Processing Systems, pages 147-155, 2019.

[17] Hengduo Li, Zuxuan Wu, Chen Zhu, Caiming Xiong, Richard Socher, and Larry S Davis. Learning from noisy anchors for one-stage object detection. In Proceedings of the IEEE/CVF Conference on Computer Vision and Pattern Recognition, pages 10588-10597, 2020.

[18] Benjin Zhu, Jianfeng Wang, Zhengkai Jiang, Fuhang Zong, Songtao Liu, Zeming Li, and Jian Sun. Autoassign: Differentiable label assignment for dense object detection. arXiv preprint arXiv:2007.03496, 2020.

[19] Zheng Ge, Songtao Liu, Zeming Li, Osamu Yoshie, and Jian Sun. Ota: Optimal transport assignment for object detection. In CVPR, 2021.

[20] Kaiwen Duan, Song Bai, Lingxi Xie, Honggang Qi, Qingming Huang, and Qi Tian. Centernet: Keypoint triplets for object detection. In Proceedings of the IEEE International Conference on Computer Vision, pages 6569-6578, 2019.

[21] Ashish Vaswani, Noam Shazeer, Niki Parmar, Jakob Uszkoreit, Llion Jones, Aidan N Gomez, Łukasz Kaiser, and Illia Polosukhin. Attention is all you need. In Advances in neural information processing systems, pages 5998-6008, 2017.

[22] Hamid Rezatofighi, Nathan Tsoi, JunYoung Gwak, AmirSadeghian, Ian Reid, and Silvio Savarese. Generalized intersection over union: A metric and a loss for bounding box regression. In CVPR, 2019.

[23] Tsung-Yi Lin, Michael Maire, Serge Belongie, James Hays, Pietro Perona, Deva Ramanan, Piotr Dollar, and C Lawrence ' Zitnick. Microsoft coco: Common objects in context. In European conference on computer vision, pages 740-755. Springer, 2014.

[24] Kaiming He, Georgia Gkioxari, Piotr Dollar, and Ross Gir-shick. Mask r-cnn. In Proceedings of the IEEE international conference on computer vision, pages 2961-2969, 2017.

[25] Tsung-Yi Lin, Piotr Dollar, Ross Girshick, Kaiming He, Bharath Hariharan, and Serge Belongie. Feature pyramid networks for object detection. In Proceedings of the IEEE conference on computer vision and pattern recognition, pages 2117-2125, 2017.

[26] Jiaxi Wu, Songtao Liu, Di Huang, and Yunhong Wang. Multi-scale positive sample refinement for few-shot object detection. In European Conference on Computer Vision, pages 456-472. Springer, 2020.

[27] Yangtao Zheng, Di Huang, Songtao Liu, and Yunhong Wang. Cross-domain object detection through coarse-tofine feature adaptation. In Proceedings of the IEEE/CVF Conference on Computer Vision and Pattern Recognition, pages 13766-13775, 2020.

[28] Lin Song, Yanwei Li, Zhengkai Jiang, Zeming Li, Xiangyu Zhang, Hongbin Sun, Jian Sun, and Nanning Zheng. Rethinking learnable tree filter for generic 
feature transform. arXiv preprint arXiv:2012.03482, 2020.

[29] Joseph Redmon and Ali Farhadi. Yolo9000: better, faster, stronger. In Proceedings of the IEEE conference on computer vision and pattern recognition, pages 72637271, 2017.

[30] Joseph Redmon and Ali Farhadi. Yolov3: An incremental improvement. arXiv preprint arXiv:1804.02767, 2018.

[31] Songtao Liu, Di Huang, et al. Receptive field block net for accurate and fast object detection. In Proceedings of the European Conference on Computer Vision (ECCV), pages 385-400, 2018.

[32] Hei Law and Jia Deng. Cornernet: Detecting objects as paired keypoints. In Proc. Eur. Conf. Comp. Vis., pages 734-750, 2018.

[33] Jianfeng Wang, Lin Song, Zeming Li, Hongbin Sun, Jian Sun, and Nanning Zheng. End-to-end object detection with fully convolutional network. arXiv preprint arXiv:2012.03544, 2020.

[34] Kaiming He, Xiangyu Zhang, Shaoqing Ren, and Jian Sun. Deep residual learning for image recognition. In CVPR, 2016.

[35] Ilya Loshchilov and Frank Hutter. Decoupled weight decay regularization. In International Conference on Learning Representations, 2018.

[36] Jia Deng, Wei Dong, Richard Socher, Li-Jia Li, Kai Li, and Li Fei-Fei. ImageNet: A large-scale hierarchical image database. In CVPR, 2009.

[37] Xavier Glorot and Yoshua Bengio. Understanding the difficulty of training deep feedforward neural networks. In Proceedings of the thirteenth international conference on artificial intelligence and statistics, pages 249-256, 2010.

[38] J. Redmon and A. Farhadi. Yolo9000: Better, faster, stronger. In Computer Vision and Pattern Recognition (CVPR), 2017 IEEE Conference on, pages 6517-6525. IEEE, 2017.

[39] K. He, G. Gkioxari, P. Dollár, and R. Girshick, "Mask R-CNN," in 2017 IEEE International Conference on Computer Vision (ICCV), Oct. 2017, pp. 2980-2988.

[40] P. Hurney, P. Waldron, F. Morgan, E. Jones, and M. Glavin, "Review of pedestrian detection techniques in automotive far-infrared video," IET Intel. Transport Syst., vol. 9, no. 8, pp. 824-832, 2015.

[41] HE Z W, ZHANG L. End-to-end detection and reidentification integrated net for person search. Asian Conference on Computer Vision, Perth, Australia, 349364, 2018. 